\title{
Thin-walled hollow fibers for flexible high energy density fiber-shaped supercapacitors
}

\author{
Chuan He ${ }^{1,2}$, Jianli Cheng ${ }^{1}$, Yuhang Liu', Xicui Zhang ${ }^{3}$, Bin Wang ${ }^{1,2}$ \\ ${ }^{1}$ Institute of Chemical Materials, China Academy of Engineering Physics, Mianyang 621900, Sichuan, China. \\ ${ }^{2}$ Institute of Fundamental and Frontier Sciences, Yangtze Delta Region Institute (Huzhou), University of Electronic Sciences and \\ Technology of China, Chengdu 611731, Sichuan, China. \\ ${ }^{3}$ Sichuan Institute of Product Quality Supervision \& Inspection, Chengdu 610100, Sichuan, China.
}

Correspondence to: Prof. Xicui Zhang, Sichuan Institute of Product Quality Supervision \& Inspection, No.16 XingMao Street, Chengdu 610100, Sichuan, China. E-mail: zhangxicui216@163.com; Prof. Bin Wang, Institute of Fundamental and Frontier Sciences, Yangtze Delta Region Institute (Huzhou), University of Electronic Sciences and Technology of China, No. 2006 Xiyuan Avenue, Chengdu 611731, Sichuan, China. E-mail: binwang@uestc.edu.cn

How to cite this article: He C, Cheng J, Liu Y, Zhang X, Wang B. Thin-walled hollow fibers for flexible high energy density fibershaped supercapacitors. Energy Mater 2021;1:100010. https://dx.doi.org/10.20517/energymater.2021.14

Received: 23 Sep 2021 First Decision: 18 Oct 2021 Revised: 25 Oct 2021 Accepted: 26 Oct 2021 Published: 30 Oct 2021

Academic Editor: Yuping Wu Copy Editor: Yue-Yue Zhang Production Editor: Yue-Yue Zhang

\begin{abstract}
Fiber-shaped supercapacitors, which occupy minimal volume and possess remarkable flexibility, are particularly promising candidates for next-generation smart wearable devices. However, the state-of-the-art energy density and mechanical properties of fiber-shaped electrodes are far from satisfactory. Herein, hollow poly (3,4ethylenedioxythiophene):polystyrene sulfonate thin-walled fibers (HPFs) are continuously prepared by coaxial wet-spinning. These HPFs combine a simple and high continuous preparation with high electrochemical performance and flexibility, owing to their hollow nature, small diameter $(125 \mu \mathrm{m})$ and thin wall structure $(8 \mu \mathrm{m})$. As a result, the HPFs display a specific areal capacitance of $115.2 \mathrm{mF} \mathrm{cm}^{-2}$ at a current density of $0.3 \mathrm{~mA} \mathrm{~cm}$ with a high energy density of $9 \mu \mathrm{Wh} \mathrm{cm}{ }^{-2}$ at a power density of $0.112 \mathrm{~mW} \mathrm{~cm}^{-2}$. Furthermore, the HPFs maintain $81 \%$ of the initial capacitance after 10,000 cycles with $~ 100 \%$ Coulombic efficiency. More importantly, the specific capacitance is almost completely maintained after bending 3000 times at $180^{\circ}$.
\end{abstract}

Keywords: Fiber-shaped supercapacitors, PEDOT:PSS, energy density, flexible 


\section{INTRODUCTION}

With the emergence of fifth-generation wireless technology, flexible electronic devices and smart textiles hold significant potential for the next technological revolution by providing flexible, integrated and diverse applications in areas such as emergency and health management, smart homes and surveillance ${ }^{[1-4]}$. Among the different components of wearable devices, energy storage devices, such as supercapacitors, are one of the vital components and they need to meet the flexibility, wearability and high electrochemical performance requirements for application in flexible electronic devices. Traditional energy storage devices, such as symmetrical capacitors and supercapacitors, play an important role in human daily life. However, they cannot meet the flexibility and wearability demands of flexible electronic devices, owing to their intrinsically stiff structure ${ }^{[5,6]}$.

Inspired by textile clothing fibers, fiber-shaped electrodes have become a hot topic because of their intrinsic flexibility, easy integration into textiles and facile preparation ${ }^{[7-10]}$. Conductive polymers, such as poly $(3,4-$ ethylenedioxythiophene):polystyrene sulfonate (PEDOT:PSS), can simultaneously combine electrode materials with high conductivity. Furthermore, PEDOT:PSS polymers are also commercially available with outstanding aqueous distribution performance. More importantly, PEDOT:PSS electrodes, with the advantages of fast charge-discharge capability, one-dimensional intrinsic flexibility and superb rate performance ${ }^{[11]}$, represent ideal alternatives for preparing flexible supercapacitor devices. For example, Yuan prepared meter-long PEDOT:PSS fibers with good mechanical properties. The prepared PEDOT:PSS fibers could be easily woven, sewed, knotted and braided as a fiber-shaped yarn supercapacitor electrode with an areal energy density of $4.13 \mu \mathrm{Wh} \mathrm{cm} \mathrm{cm}^{-2}$ and robust performance (strength strain of $85 \mathrm{MPa}$ ) under repeated bending tests ${ }^{[12]}$. Wang reported a highly crystalline "all-in-one" PEDOT:PSS fiber electrode with a high electrical conductivity of $1771.8 \mathrm{~S} \mathrm{~cm}^{-1}$ and a tensile strength of $112.7 \mathrm{MPa}$. The assembled eight seriesconnected supercapacitor groups displayed an energy density of $41.1 \mu \mathrm{Wh} \mathrm{cm} \mathrm{cm}^{-2}$ at a power density of $3520 \mathrm{~mW} \mathrm{~cm}^{-2}$ and without significant capacitance degradation after stretching up to $400 \%{ }^{[13]}$. These works highlight the potential of PEDOT:PSS fiber-shaped electrodes for flexible electronic devices.

Nevertheless, PEDOT:PSS fiber electrodes are known to be solid structures with relatively low capacitance and limited specific energy density, owing to their low effective specific surface area (E-SSA), which refers to the actual electrolyte contact area ${ }^{[14]}$. It is well known that the adsorption of electrolyte ions inside solid fibers is very limited, primarily due to the difficulty of ion diffusion along the radial direction of the fiber electrode $^{[15]}$. Therefore, efficiently improving the E-SSA of a fiber electrode is one of the most urgent challenges for achieving high energy density. Qu et al ${ }^{[16]}$ proposed a confined hydrothermal method that depends on the gas released during graphene oxide (GO) reduction to fabricate reduced graphene oxide (rGO)/PEDOT:PSS hollow composite fibers (HCFs). In addition, the areal capacitance of HCFs has a significant improvement of $60 \%$ compared to the solid composite fibers because of the hollow interior. However, this method cannot realize continuous preparation because of the limited length of capillary glass tubes. Furthermore, the uniformity of hollow structures requires precise control of the experimental conditions. Therefore, combining simple and high operability continuous wet-spinning with the hollow structure concept may be an effective strategy to improve the energy density and mechanical properties simultaneously.

In this work, thin-walled hollow PEDOT:PSS fiber electrodes with high flexibility and electrical conductivity are prepared by rational design via coaxial wet-spinning. The prepared fiber electrodes show good flexibility with a high tensile strength of $383 \mathrm{MPa}$ at a large breaking strain of $25 \%$ and a high electrical conductivity of $362.8 \mathrm{~S} \mathrm{~cm}^{-1}$ (1514 S cm $\mathrm{cm}^{-1}$ calculated by excluding the hollow interior volume). In addition, the fiber electrodes can be rolled up and woven into textiles without any obvious electrochemical performance decay. 
When used as electrode materials for gel electrolyte fiber-shaped supercapacitors (FSCs), they show a wide

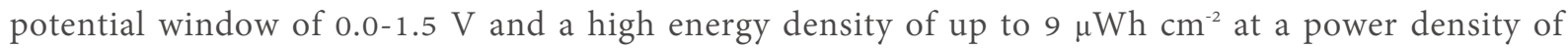
$0.112 \mathrm{~mW} \mathrm{~cm}^{-2}$. Furthermore, the specific capacitance remains relatively unaffected after bending 3000 times and can maintain an initial specific capacitance of $81 \%$ after 10000 cycles. This excellent electrochemical performance can be attributed to the unique hollow structure, stable gel electrolyte and unique electrochemical characteristics of the PEDOT:PSS fibers. This work provides an alternative method for the design of flexible FSC systems with promising performance for applications in wearable and flexible electronics.

\section{EXPERIMENTAL}

\section{Materials}

Clevios PH1000 (PEDOT:PSS, 1.0-1.3 wt.\%) was purchased from HC Starck, Inc. Lithium chloride (LiCl, AR), polyvinyl alcohol (PVA, AR) 1788 ( $\mathrm{Mw} 75,000-78,000)$ and methanol $(\mathrm{MeOH}, \mathrm{AR})$ were provided by Aladdin. $\mathrm{CaCl}_{2}(\mathrm{AR})$ and concentrated $\mathrm{H}_{2} \mathrm{SO}_{4}(\geq 98 \%)$ were purchased from Kelong Chemical Reagent Company.

\section{Synthesis of hollow and solid PEDOT:PSS fibers by wet-spinning}

The PEDOT:PSS was concentrated at $2.0 \mathrm{wt} . \%$ by evaporating water at $50{ }^{\circ} \mathrm{C}$. Two microinjection pumps injected the coagulation solution and the concentrated PEDOT:PSS from different syringes into the coagulation solution with $\mathrm{CaCl}_{2}(2.5 \mathrm{wt}$.\%) which consists of a water:ethanol solution with a volume ratio of $1: 3$ at extruded velocities of 3.0 and $1.5 \mathrm{~min} / \mathrm{mL}$, respectively. The sizes of the coaxial needles used in the wet-spinning were $18 \mathrm{G}$ and $27 \mathrm{G}$. The hollow PEDOT:PSS fibers (HPFs) were fully cross-linked in the coagulation bath for $30 \mathrm{~min}$ to maintain their morphology. They were then washed by deionized water and immersed in the $\mathrm{MeOH}$ solvent for 30 min to remove the long PSS chains from the surfaces of the fibers. Subsequently, the HPFs were dried on a polytetrafluoroethylene plate at $90{ }^{\circ} \mathrm{C}$ for $10 \mathrm{~min}$. Finally, the HPFs were immersed in concentrated sulfuric acid within $30 \mathrm{~min}$ and sufficiently washed by deionized water to remove part of the insulated PSS. The HPFs were then dried in an oven at $120^{\circ} \mathrm{C}$ for $10 \mathrm{~min}$ to remove the residual water. The preparation conditions for the solid PEDOT:PSS fibers (SPFs) were similar but without pumping of the coagulation bath.

\section{Preparation of gel electrolyte and assembly of gel electrolyte FSCs}

First, a 3.5 M LiCl solution was prepared and stirred by a magnetic stirrer until it was completely dissolved. Second, $3 \mathrm{~g}$ of PVA were dissolved in a $30 \mathrm{~mL} \mathrm{LiCl}$ solution at $80^{\circ} \mathrm{C}$. Finally, it was continually stirred until the gel solution became clear. For the FSCs, two HPFs or SPFs were placed side by side in parallel and covered with the PVA-LiCl gel electrolyte on the surface. Both ends of the fibers were fixed to the glass slide using conductive tapes and silver adhesives.

\section{Characterization and electrochemical tests}

The surface morphologies of the HPFs and SPFs were measured by scanning electron microscopy (SEM, Ultra 55, Carl Zeiss SMT Pte Ltd.). The strain-stress curves of the HPFs and SPFs were measured by a microcomputer controlled electronic universal material testing machine (HY-0350, Shanghai Heng Wing Precision Instrument Co., Ltd.). The Fourier transform infrared spectra were measured at a scanning range from 4000 to $500 \mathrm{~cm}^{-1}$ [WQF-530, Beijing Beifen-Ruili Analytical Instrument (Group) Co., Ltd.]. X-ray diffraction (XRD) was measured by a Bruker D2 diffractometer using $\mathrm{Cu}$ Ka radiation at a wavelength of $1.5406 \AA$ A Cyclic voltammetry (CV) with scan rates from 20 to $200 \mathrm{mV} \mathrm{s}^{-1}$ and electrochemical impedance spectroscopy (EIS) in a frequency range of $200 \mathrm{kHz}$ to $100 \mathrm{mHz}$ at $0 \mathrm{~V}$ bias with an $\mathrm{AC}$ amplitude of $10 \mathrm{mV}$ were measured using a VSP-300 electrochemical workstation (Bio-Logic SAS, France). Furthermore, the conductivity of the fiber electrodes was obtained by linear scanning voltammetry (LSV) at a scan rate of 5 
$\mathrm{mV} \mathrm{s}^{-1}$ in the range of 0-1 V using the VSP-300 electrochemical workstation. Galvanostatic charge-discharge (GCD) curves were recorded by an Arbin Instruments testing system (BT-2000, Arbin) at a current density of $2 \mathrm{~mA} \mathrm{~cm} \mathrm{~cm}^{-2}$.

\section{RESULTS AND DISCUSSION}

In order to compare the morphological differences, different fibers with or without the hollow structure (HPFs and SPFs) were prepared using similar experimental conditions and characterized. Figure 1A illustrates the fabrication procedure, the microstructure of the hollow coaxial fibers and the charge distribution on the internal and external surfaces. In addition, a continuously hollow HPF with a length of $\sim 0.7 \mathrm{~m}$ was prepared by wet-spinning, as shown in Supplementary Figure 1, which demonstrates that the method has the potential for continuous scale preparation. The microscopic morphologies of the HPFs and SPFs were characterized by SEM, as displayed in Figure 1B-E. It can be clearly observed in Figure 1B that the prepared fibers showed a thin-walled structure with a hollow and uniform structure in the fiber center. The diameter of the HPFs was $\sim 125 \mu \mathrm{m}$ and the wall thickness was only $8 \mu \mathrm{m}$, which depends on the different extruded velocities of the concentrated PEDOT:PSS solution and coagulation bath. Interestingly, the thin-walled hollow structure could be well maintained without structure collapse under solvent and high-temperature treatment, thereby demonstrating desirable structural stability.

In comparison, the cross-sectional structure of the SPFs can also be observed from Figure $1 \mathrm{C}$, which demonstrates the solid structure of the prepared PEDOT:PSS fibers with a diameter of $\sim 100 \mu \mathrm{m}$. The diameter of the SPFs was smaller than that of the HPFs. The phenomenon of smaller diameters of the SPFs could be ascribed to two reasons. First, without the internal coagulation bath, the external PEDOT:PSS may lead to self-healing during wet-spinning. Second, the interior solid structure makes a greater contribution to the volume shrinkage of the SPFs during evaporation. Furthermore, the side-view SEM images of the HPFs and SPFs are demonstrated in Figure 1D and E. The diameter of these conductive fibers was uniform, which illustrates the structural stability of the polymer fibers prepared from coaxial wet-spinning. The morphological differences between the SPFs and HPFs can also be clearly observed. With similar experimental conditions, the HPFs demonstrate relatively smooth surfaces compared to the SPFs, owing to an even multi-step solvent treatment and water evaporation process from the internal and external surfaces simultaneously.

To better understand their mechanical performance, the strain-stress curves of the HPFs and SPFs were characterized, as shown in Figure 2A. Interestingly, the tensile strength of the thin-walled HPFs showed a noticeable increase of $87 \%$ to $384 \mathrm{MPa}$ compared with that of the SPFs (205 MPa) under the same strain of $25 \%$. The increased tensile strength of the HPFs may be caused by the relatively complete treatment of $\mathrm{H}_{2} \mathrm{SO}_{4}$ and the stronger $\pi-\pi$ interactions of PEDOT. Therefore, it can be anticipated that the prepared hollow one-dimensional fibrous structure benefits the improvement of the mechanical characteristics and specific capacitance per mass of the fiber electrodes. Figure $2 \mathrm{~B}$ shows the LSV curves of the SPFs and HPFs at a scanning rate of $5 \mathrm{mV} \mathrm{s}^{-1}$. The conductivity of the SPFs and HPFs could be calculated by equation (1):

$$
\sigma=\frac{L}{R S}
$$

where $L, R$ and $S$ are the length, resistance and cross-section area of the fiber electrodes, respectively. 
A

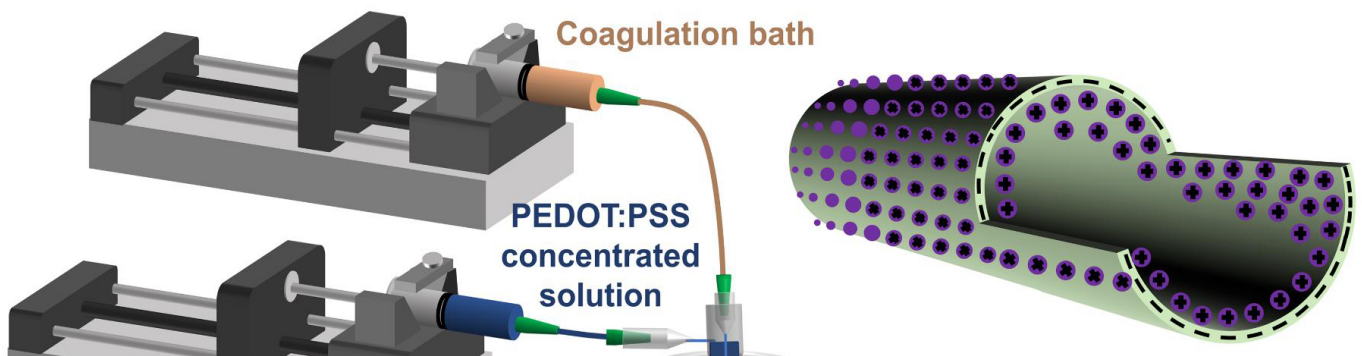

$\oplus$ Positive charge
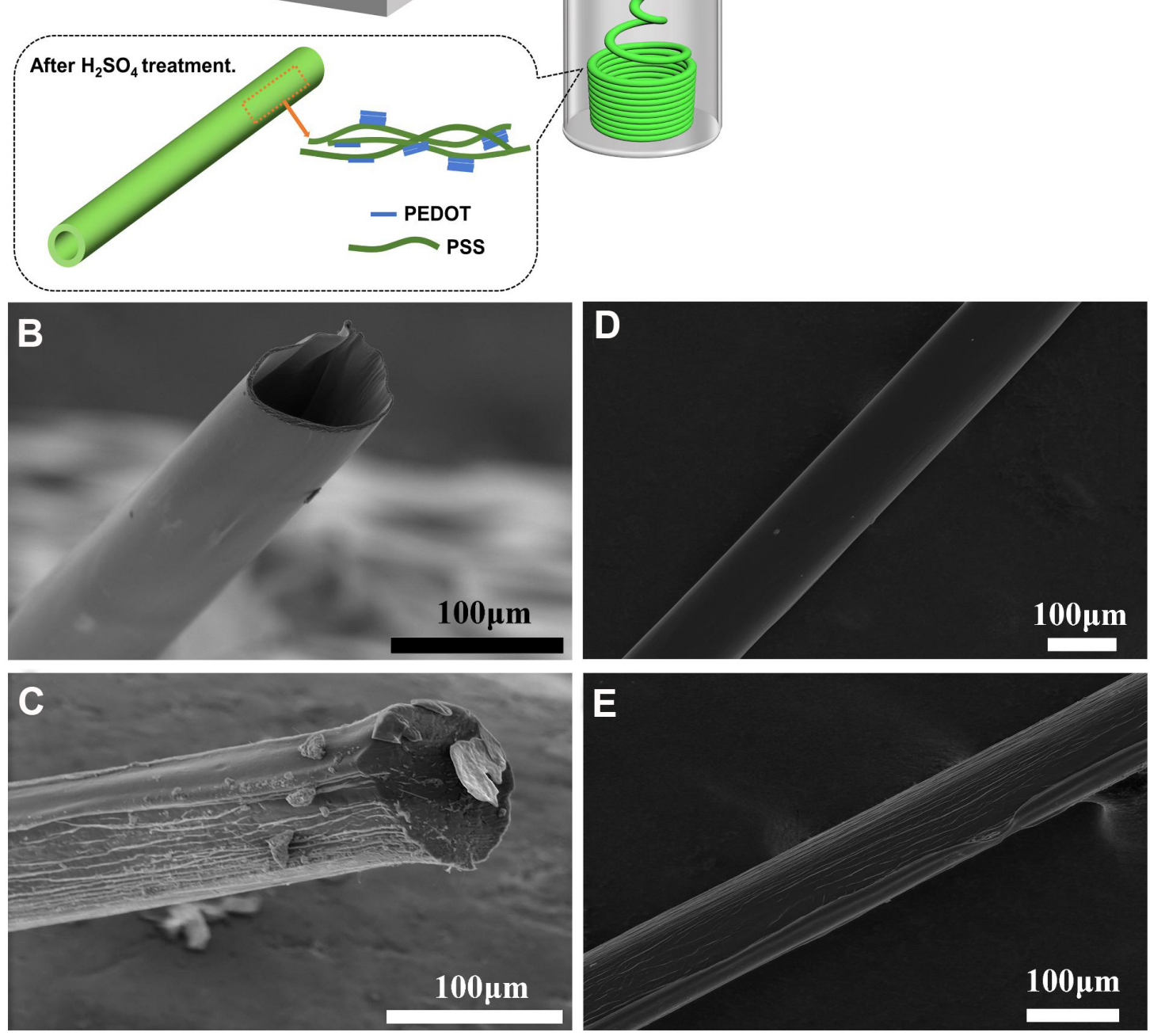

Figure 1. (A) Schematic illustration of preparation process, microstructure and surface charge distribution of HPFs. Cross-sectional SEM images of (B) HPFs and (C) SPFs. Side-view SEM images of (D) HPFs and (E) SPFs. HPF: Hollow poly(3,4ethylenedioxythiophene):polystyrene sulfonate thin-walled fibers; SPF: solid PEDOT:PSS fibers; SEM: scanning electron microscopy.

The HPFs displayed an electrical conductivity of $1514 \mathrm{~S} \mathrm{~cm}^{-1}$ calculated by excluding the area of the hollow interior. In contrast, the conductivity of the SPFs was $421 \mathrm{~S} \mathrm{~cm}^{-1}$, which is $\sim 25 \%$ that of the HPFs. Moreover, the electrochemical performance of the HPFs and SPFs were also measured by EIS, with the results shown in Figure $2 \mathrm{C}$ for a frequency range of $200 \mathrm{kHz}$ to $100 \mathrm{mHz}$. In the high-frequency region, the $\mathrm{X}$-intercept of the Nyquist plot represents the equivalent series resistance (ESR), corresponding to the electrolyte 
A
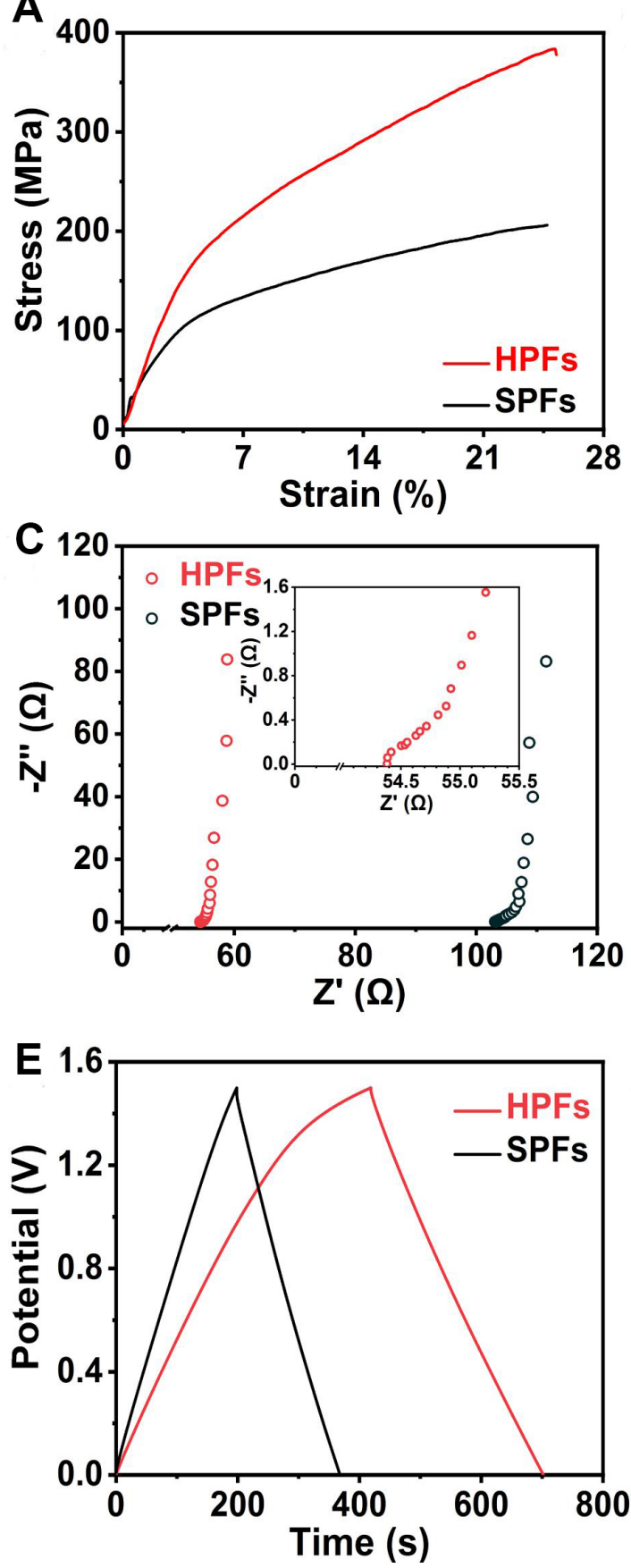
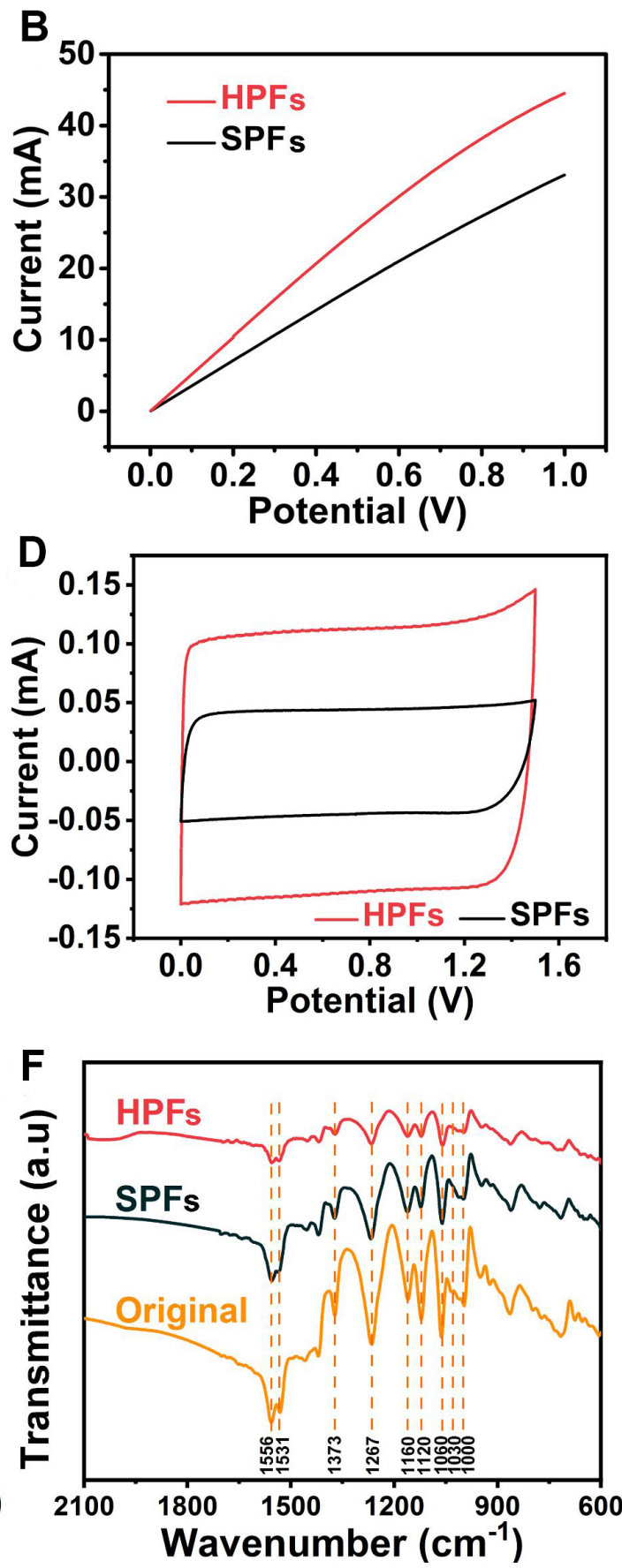

Figure 2. (A) Strain-stress curves of HPFs and SPFs. (B) LSV curves of HPFs and SPFs at a scanning rate of $5 \mathrm{mV} \mathrm{s}^{-1}$. (C) EIS analysis of HPFs and SPFs (inset shows the magnified spectrum of HPFs). (D) CV and (E) GCD curves of HPFs and SPFs at 0.0-1.5 V. (F) Fourier transform infrared spectra of original polymer fibers without $\mathrm{H}_{2} \mathrm{SO}_{4}$ treatment, SPFs and HPFs. HPF: Hollow poly (3,4ethylenedioxythiophene):polystyrene sulfonate thin-walled fibers; SPF: solid PEDOT:PSS fibers; LSV: linear scanning voltammetry; EIS: electrochemical impedance spectroscopy; CV: cyclic voltammetry; GCD: galvanostatic charge-discharge.

resistance, internal resistance of the electrode and the contact resistance. The HPF electrode showed a smaller ESR of $54.4 \Omega$ in the high-frequency range in comparison to the ESR of the SPFs $(104 \Omega)$. This result may be due to the relative difficulty in removing part of the insulated PSS unit in the solid structure ${ }^{[17-20]}$. Obviously, the Nyquist plot shows only one semicircle, corresponding to the charge transfer at the HPF or 
SPF electrode/electrolyte interfaces ${ }^{[21]}$. Compared with the HPFs, the SPFs displayed a larger semicircle, illustrating that SPFs had a larger charge transfer resistance. These results are in good agreement with the LSV results. In the low-frequency region, the vertical degree of the sloped line showed the corresponding characteristics of pure capacitive behavior. The HPFs showed a more vertical line than the SPFs at low frequency, thereby demonstrating faster ion diffusion.

To further investigate the influence of the hollow structure of the HPFs on their electrochemical properties, the CV and GCD characterization of the assembled FSCs based on HPFs or SPFs was carried out using a 3.5 M PVA-LiCl gel electrolyte in a potential window of 0.0-1.5 V, as shown in Figure $2 \mathrm{D}$ and E. The CV curves of the SPFs and HPFs are characterized in Figure $2 \mathrm{D}$ with a scanning rate of $20 \mathrm{mV} \mathrm{s}^{-1}$. It can be seen that the area of the rectangular curve for the HPFs was much larger than for the SPFs, demonstrating that the HPFs showed better charge storage capability. Comparing the specific mass capacity $\left(\mathrm{C}_{\mathrm{m}}\right)$ of the HPFs and SPFs, the $\mathrm{C}_{\mathrm{m}}$ of the HPFs $\left(173.8 \mathrm{~F} \mathrm{~g}^{-1}\right.$ ) was $\sim 5.4$ times higher than that of the SPFs. The specific areal capacitances of the HPFs and SPFs were calculated to be 112.7 and $67.5 \mathrm{mF} \mathrm{cm}^{-2}$, respectively. Compared to the SPFs, the HPFs exhibited a distinct capacitance increase of $\sim 67 \%$. Furthermore, the discharge times of the HPF and SPF electrodes were 288 and 170 s, respectively. Notably, the GCD curves of the HPF-based FSCs showed a symmetric shape up to $1.5 \mathrm{~V}$ with a small IR drop (0.008 V). However, the IR drop of the SPFs was $0.025 \mathrm{~V}$, which was three times that of the HPFs, implying the low conductivity of the SPFs. Therefore, comparing the electrochemical and mechanical test results of the HPFs and SPFs, the HPFs showed higher specific capacitance and better mechanical performance.

This exceptional performance may be derived from the following processes. First, the $\mathrm{HSO}_{4}^{-}$ions yielded by two $\mathrm{H}_{2} \mathrm{SO}_{4}$ molecules can partially replace the negatively charged PSS, which is not coupled with PEDOT according to equation (2):

$$
\mathrm{H}_{2} \mathrm{SO}_{4}+\mathrm{PSS}^{-} \rightarrow \mathrm{HSO}_{4}^{-}+\mathrm{PSSH}
$$

PEDOT:PSS is then rearranged under the $\pi-\pi$ stacking to form dense PEDOT networks with significant morphological and crystalline structure change ${ }^{[18,22]}$. Simultaneously, the thin-walled hollow structure of the HPFs facilitates, thorough the solvent treatment and dense conductive network, rapid ion transport and increases the interfacial area of the electrode and electrolytes, leading to increased specific capacitance. To verify this assumption, the attenuated total reflectance-Fourier transform infrared spectroscopy spectra were measured for the SPFs without $\mathrm{H}_{2} \mathrm{SO}_{4}$ treatment, SPFs and HPFs, as demonstrated in Figure $2 \mathrm{~F}$. Several peaks located at $1267,1373,1531$ and $1556 \mathrm{~cm}^{-1}$ could be attributed to the C-C or C=C stretching of the quinoidal structure and the ring stretching of the thiophene ring of PEDOT:PSS, respectively. The characteristic peaks of $-\mathrm{SO}_{3} \mathrm{H}$ in PEDOT:PSS were located at $1060 \mathrm{~cm}^{-1}$ and the absorbance peaks located at $1000,1030,1120$ and $1160 \mathrm{~cm}^{-1}$ corresponded to the asymmetric and symmetric stretching vibration of the $\mathrm{SO}_{3}{ }^{-}$group of the PSS, respectively ${ }^{[23-25]}$. Furthermore, compared with that of the pristine SPFs, all of the intensities of these PSS characteristic peaks significantly decreased for the SPFs and HPFs after the sulfuric acid treatment, demonstrating the partial removal of the PSS after treatment.

To characterize the electrochemical properties of the HPFs, the FSCs were prepared by two HPFs placed in parallel using the 3.5 M PVA-LiCl gel electrolyte. Figure 3A shows the CV curves of the HPF electrode measured at different potential windows at a scan rate of $20 \mathrm{mV} \mathrm{s}^{-1}$. It can be observed that there was no obvious polarization when the potential reached $1.5 \mathrm{~V}$. The CV curves maintained their quasi-rectangular shape at $1.5 \mathrm{~V}$, which is consistent with the results of the potential window tested by GCD at a current 

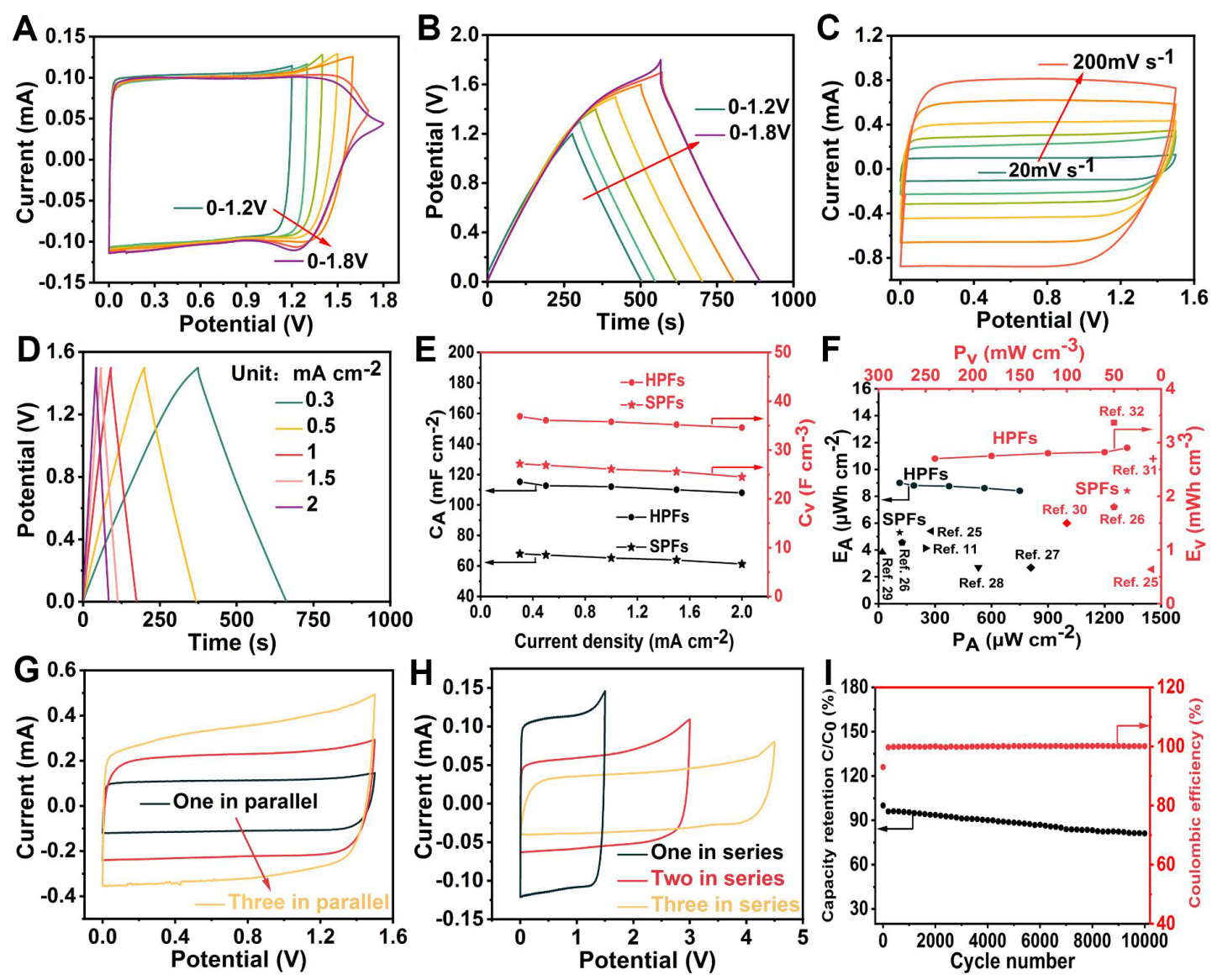

Figure 3. (A) CV and (B) GCD curves of HPFs tested at different potential windows from 0.0 to 1.2-1.8 V. (C) CV curves of HPFs at different scan rates. (D) GCD curves of HPFs at different current densities and (E) rate capabilities of HPFs and SPFs. (F) Ragone plots of HPFs compared with SPFs and other reported results. CV curves of one, two and three devices connected $(G)$ in parallel and $(H)$ in series at a scan rate of $20 \mathrm{mV} \mathrm{s}^{-1}$. (I) Cycling performance of FSCs based on HPFs measured at a current density of $2 \mathrm{~mA} \mathrm{~cm}^{-2}$. CV: Cyclic voltammetry; GCD: galvanostatic charge-discharge; HPF: hollow poly(3,4-ethylenedioxythiophene):polystyrene sulfonate thinwalled fibers; FSC: fiber-shaped supercapacitor; SPF: solid PEDOT:PSS fibers.

density of $0.3 \mathrm{~mA} \mathrm{~cm}^{-2}$ [Figure 3B]. For potentials of $>1.5 \mathrm{~V}$, the potential polarization increased distinctly. Therefore, the potential window of 0.0-1.5 V was chosen for the subsequent tests of the FSCs. In addition, the CV curves also showed typical rectangular shapes and obviously increased current response values with the scan rate increased from 20 to $200 \mathrm{mV} \mathrm{s}^{-1}$, revealing the fast charge-discharge capability [Figure $3 \mathrm{C}$ ].

Furthermore, the GCD curves of the HPFs showed outstanding symmetry, as shown in Figure 3D, further verifying the excellent capacitive characteristics. With increasing applied current density, the corresponding discharge time and specific capacitance gradually decreased [Figure 3E]. No obvious IR drop was observed at the beginning process of the constant current discharge, indicative of low internal resistance. Tested at a current density of $0.3 \mathrm{~mA} \mathrm{~cm}^{-2}$, the specific areal capacitance of the HPFs reached $115.2 \mathrm{mF} \mathrm{cm}^{-2}$, corresponding to a mass capacity of $173.8 \mathrm{~F} \mathrm{~g} \mathrm{~g}^{-1}$ and a volumetric capacitance $\left(\mathrm{C}_{\mathrm{V}} \mathbb{\nabla}\right.$ of $36.87 \mathrm{~F} \mathrm{~cm}^{-3}$, while the corresponding values of the SPFs were $68 \mathrm{mF} \mathrm{cm}^{-2}, 25.9 \mathrm{~F} \mathrm{~g}^{-1}$ and $27.2 \mathrm{~F} \mathrm{~cm}^{-3}$, respectively. With the current densities further increased to 1.5 and $2.0 \mathrm{~mA} \mathrm{~cm}^{-2}$, the HPFs still maintained high areal capacitances of 110 and $108 \mathrm{mF} \mathrm{cm}^{-2}$ (35.2 and $34.6 \mathrm{~F} \mathrm{~cm}^{-3}$ for $\mathrm{C}_{\mathrm{v}}$ or 169 and $163 \mathrm{~F} \mathrm{~g}^{-1}$ for $\mathrm{C}_{\mathrm{m}}$ ), respectively, demonstrating their excellent rate capability and high specific capacitance retention even at very high current densities. 
The Ragone plot of the HPF-based FSCs was further calculated, as shown in Figure 3F. to compare the areal energy density $\left(\mathrm{E}_{\mathrm{A}}\right)$ and volumetric energy density $\left(\mathrm{E}_{\mathrm{V}}\right)$ with those of the SPF-based FSCs and previously

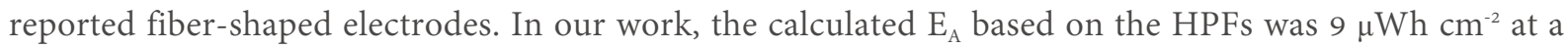

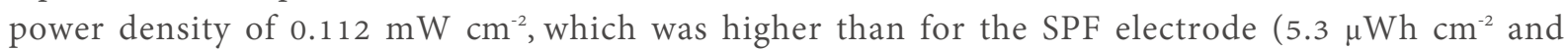

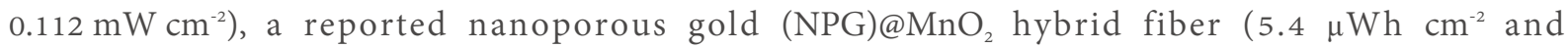
$\left.0.28 \mathrm{~mW} \mathrm{~cm}^{-2}\right)^{[26]}$, a highly-wrinkled rGO-PEDOT:PSS fiber $\left(4.55 \mu \mathrm{Wh} \mathrm{cm}^{-2} \text { and } 0.125 \mathrm{~mW} \mathrm{~cm}^{-2}\right)^{[27]}$ and a TiN@C fiber $\left(2.68 \mu \mathrm{Wh} \mathrm{cm}^{-2} \text { and } 0.809 \mathrm{~mW} \mathrm{~cm}^{-2}\right)^{[28]}$. In addition, the $\mathrm{E}_{\mathrm{A}}$ found in this study was also better than for asymmetric FSCs supported by carbon fibers coated by $\mathrm{MnO}_{2}\left(\mathrm{CF} / \mathrm{MnO}_{2}\right)$ and $\mathrm{MoO}_{3}\left(\mathrm{CF} / \mathrm{MoO}_{3}\right)$ $\left(2.7 \mu \mathrm{Wh} \mathrm{cm}{ }^{-2} \text { and } 0.53 \mathrm{~mW} \mathrm{~cm}^{-2}\right)^{[29]}$, coaxial fibers electrodes with a mixture of $\mathrm{rGO}$ and carbon nanotube (CNT) core and sodium carboxymethyl cellulose sheath $\left(3.84 \mu \mathrm{Wh} \mathrm{cm}^{-2} \text { and } 0.02 \mathrm{~mW} \mathrm{~cm}^{-2}\right)^{[30]}$ and PEDOT:PSS fibers $\left(4.13 \mu \mathrm{Wh} \mathrm{cm}^{-2} \text { and } 0.25 \mathrm{~mW} \mathrm{~cm}^{-2}\right)^{[12]}$. The HPF-based FSCs delivered an $\mathrm{E}_{\mathrm{V}}$ of $2.9 \mathrm{mWh}$ $\mathrm{cm}^{-3}$ at a power density of $36 \mathrm{~mW} \mathrm{~cm}^{-3}$, which exceeded the SPFs $\left(2.1 \mathrm{mWh} \mathrm{cm}^{-3}\right.$ and $\left.45 \mathrm{~mW} \mathrm{~cm} \mathrm{~cm}^{-3}\right)$, NPG@ $\mathrm{MnO}_{2}$ hybrid fibers $\left(0.64 \mathrm{mWh} \mathrm{cm}^{-3} \text { and } 10 \mathrm{~mW} \mathrm{~cm}^{-3}\right)^{[26]}$, highly-wrinkled rGO-PEDOT:PSS fibers (1.8 $\mathrm{mWh} \mathrm{cm}^{-3}$ and $\left.50 \mathrm{~mW} \mathrm{~cm}^{-3}\right)^{[27]}$, asymmetric FSCs fabricated by multi-walled carbon nanotubes with $\mathrm{MoS}_{2}$ and rGO nanosheets (MWCNT/MoS - -rGO) as anodes and rGO/MWCNTs as cathodes $\left(1.5 \mathrm{mWh} \mathrm{cm}^{-3}\right.$ and

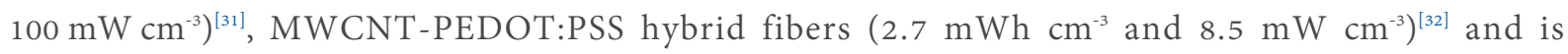
comparable to that of CNT-rGO@ $\mathrm{MnO}_{2}$ fibers $\left(3.37 \mathrm{mWh} \mathrm{cm}^{-3} \text { and } 50 \mathrm{~mW} \mathrm{~cm}^{-3}\right)^{[33]}$.

To demonstrate their capability to adapt to the different practical application requirements of devices, different numbers of FSCs based on HPFs were connected in series or parallel, as shown in Figure $3 \mathrm{G}$ and $\mathrm{H}$. The voltage or current of the devices can be increased severalfold with the number of connected devices in series or parallel. As a result, the voltages showed a monotonous linear increase of $1.5,3.0$ and $4.5 \mathrm{~V}$ by increasing the number of serial cells from one to three, respectively. Similarly, the current increased with an increased number of parallel-connected devices, demonstrating excellent performance uniformity. Moreover, the long-term cycle stability of the FSCs based on HPFs was evaluated at a current density of $2 \mathrm{~mA} \mathrm{~cm}^{-2}$. As shown in Figure 3I, the specific capacitance retention after 10,000 cycles was higher than $81 \%$, suggesting that the device exhibited impressive stability over long cycling. More importantly, the Coulombic efficiency was maintained close to $100 \%$, indicating high charge utilization. As shown in Supplementary Figure 2A, the SEM images of the HPFs after cycling do not show an obvious change of the surface or hollow structure, illustrating their good structural stability during long-term cycling. Furthermore, the HPFs after cycling showed a similar pattern with a slight decrease in the XRD intensity [Supplementary Figure 2B], further verifying their stability. The crystalline decline can be associated with the increased ESR and charge transfer resistance $\left(\mathrm{R}_{\mathrm{ct}}\right)$ shown in Supplementary Figure $2 \mathrm{C}$.

To meet the demands of practical applications, the leakage currents of the HPF- and SPF-based FSCs were analyzed by chronoamperometry at a potential of $1.5 \mathrm{~V}$ for $2500 \mathrm{~s}$. Surprisingly, the HPF-based FSCs showed an extremely low leakage current of $2.7 \mu \mathrm{A}$, which demonstrates their stability of electrochemical energy storage compared with the $5.8 \mu \mathrm{A}$ for the SPFs [Supplementary Figure 3A]. On the basis of the electrical double layer capacitance, the ions are inclined to diffuse into the electrolyte and adsorb on the surface of the fiber electrode because of the voltage and concentration differences between the electrode and electrolyte in the open circuit state. Furthermore, we performed self-discharge tests of the devices by charging them to a certain potential $(1.5 \mathrm{~V})$ and then the retained potentials were tested after a long period of rest of $10 \mathrm{~h}$ [Supplementary Figure 3B]. The HPF-based FSCs revealed a better anti-self-discharge property with an open-circuit voltage of $0.68 \mathrm{~V}$, owing to the hollow structure and low leakage current.

To demonstrate their application in flexible electronics, the HPF-based FSCs with a length of $\sim 4 \mathrm{~cm}$ were assembled in a heat shrink tube and filled with a LiCl/PVA gel electrolyte, as shown in Figure 4A. Figure 4B 

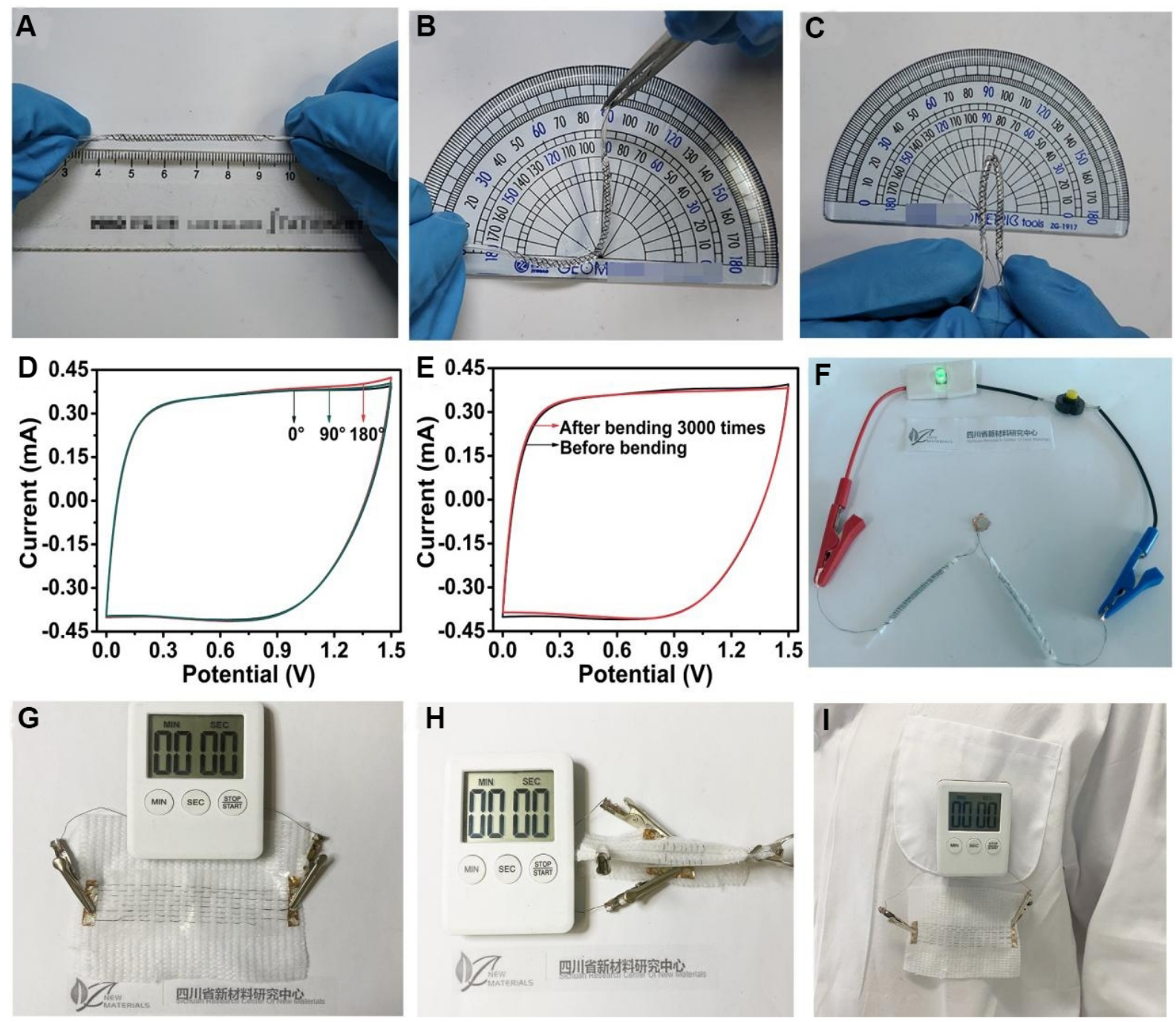

Figure 4. Photographs of HPF-based FSCs of $\sim 4 \mathrm{~cm}$ in length at the (A) straight and bent at (B) $90^{\circ}$ and (C) $180^{\circ}$ states. (D) CV curves of the FSCs bent at different angles at a scan rate of $20 \mathrm{mV} \mathrm{s}^{-1}$. (E) CV curves of FSCs before and after bending at $180^{\circ}$ for 3000 times. (F) Optical image of two individual FSCs based on HPF electrodes connected in series to light up a green LED. Fabricated textile devices integrated into the cloth with four HPFs connected in parallel to power an electronic timer at the (G) flat and (H) bending $180^{\circ}$ states. (I) Demonstration of a flexible wearable device. HPF: Hollow poly(3,4-ethylenedioxythiophene):polystyrene sulfonate thin-walled fibers; FSC: fiber-shaped supercapacitor; CV: cyclic voltammetry.

-D present the CV curves tested at various bending angles, which are well overlapped without a significant shape change, indicating good adaptability to bend at different angles. More importantly, the assembled device was bent at $180^{\circ}$ for 3000 times and the corresponding CV characterized. It can be observed from the $\mathrm{CV}$, as shown in Figure 4E, that the curves do not show a significant change after bending 3000 times, manifesting excellent electrode stability. Additionally, two individual devices connected in series after easily charging could power a green light-emitting diode with a minimum voltage requirement of $2 \mathrm{~V}$ for $\sim 8 \mathrm{~min}$ [Figure $4 \mathrm{~F}$ ]. Benefiting from the superb mechanical properties of the HPFs, textile supercapacitors were assembled by weaving them into a fabric consisting of four FSCs connected in parallel with a length of $7 \mathrm{~cm}$ [Figure $4 \mathrm{G}$ ], which can directly power a commercial electronic timer with a voltage demand of $1.5 \mathrm{~V}$ for $3 \mathrm{~min}$. The demonstrated textile supercapacitor can normally provide the power even with bending at $180^{\circ}$ for several times without an obvious performance decay, as shown in Figure $4 \mathrm{H}$ and I. The above results 
give an encouraging demonstration of these textile supercapacitor devices applied in flexible electronic devices.

\section{CONCLUSION}

In summary, hollow thin-walled PEDOT:PSS fiber electrodes with high conductivity, excellent electrochemical performance and good flexibility were prepared by wet-spinning and used as flexible electrodes to assemble high energy density FSCs and textile supercapacitors. By combining the flexibility and specific hollow structure, the HPF-based FSCs showed superb rate capability, high specific capacitance, impressive long-term cycle stability and high capacitance retention after bending 3000 times. Additionally, this exceptional performance may be attributed to the synergistic effects of the unique hollow structure, efficient PEDOT conduction network, wide electrochemical windows and increased contact areas with the electrolyte. In brief, this work provides insights into the potential application of wearable textile supercapacitor devices in flexible electronic devices.

\section{DECLARATIONS}

\section{Authors' contributions}

Wrote and reviewed the manuscript: He C, Cheng J, Liu Y, Zhang X, Wang B

\section{Availability of data and materials}

The linked data has been added in the manuscript. The raw/processed data required to reproduce these findings can be obtained from the request or the first author.

\section{Financial support and sponsorship}

The authors acknowledge the financial supports by the National Natural Science Foundation of China (No. 21875226, 52072352, U20A2072), the Foundation for the Youth S\&T Innovation Team of Sichuan Province(2020JDTD0035), Tianfu Rencai Plan, the Chengdu Talent plan, and Science and Technology Projects for Administration for Market Regulation of Sichuan Province (SCSJ2020016).

\section{Conflicts of interest}

All authors declared that there are no conflicts of interest.

\section{Ethical approval and consent to participate}

Not applicable.

\section{Consent for publication}

Not applicable.

\section{Copyright}

(c) The Author(s) 2021.

\section{REFERENCES}

1. Pan Z, Yang J, Zhang Y, Gao X, Wang J. Quasi-solid-state fiber-shaped aqueous energy storage devices: recent advances and prospects. J Mater Chem A 2020;8:6406-33. DOI

2. Wang L, Fu X, He J, et al. Application challenges in fiber and textile electronics. Adv Mater 2020;32:e1901971. DOI PubMed

3. Manjakkal L, Pullanchiyodan A, Yogeswaran N, Hosseini ES, Dahiya R. A wearable supercapacitor based on conductive PEDOT:PSS-coated cloth and a sweat electrolyte. Adv Mater 2020;32:e1907254. DOI PubMed

4. Lai Y, Lu H, Wu H, et al. Elastic multifunctional liquid-metal fibers for harvesting mechanical and electromagnetic energy and as selfpowered sensors. Adv Energy Mater 2021;11:2100411. DOI

5. Yang J, Li G, Pan Z, et al. All-solid-state high-energy asymmetric supercapacitors enabled by three-dimensional mixed-valent MnOx nanospike and graphene electrodes. ACS Appl Mater Interfaces 2015;7:22172-80. DOI PubMed

6. Zhu M, Wang Z, Li H, et al. Light-permeable, photoluminescent microbatteries embedded in the color filter of a screen. Energy 
Environ Sci 2018;11:2414-22. DOI

7. Ma W, Zhang Y, Pan S, et al. Smart fibers for energy conversion and storage. Chem Soc Rev 2021;50:7009-61. DOI PubMed

8. Lee C, Lai K, Lin C, et al. A paper-based electrode using a graphene dot/PEDOT:PSS composite for flexible solar cells. Nano Energy 2017;36:260-7. DOI

9. Song W, Fan X, Xu B, et al. All-solution-processed metal-oxide-free flexible organic solar cells with over 10\% efficiency. Adv Mater 2018;30:e1800075. DOI PubMed

10. Tian J, Cui N, Chen P, Guo K, Chen X. High-performance wearable supercapacitors based on PANI/N-CNT@CNT fiber with a designed hierarchical core-sheath structure. J Mater Chem A 2021;9:20635-44. DOI

11. Yang Z, Jia Y, Niu Y, et al. One-step wet-spinning assembly of twisting-structured graphene/carbon nanotube fiber supercapacitor. Journal of Energy Chemistry 2020;51:434-41. DOI

12. Yuan D, Li B, Cheng J, et al. Twisted yarns for fiber-shaped supercapacitors based on wetspun PEDOT:PSS fibers from aqueous coagulation. J Mater Chem A 2016;4:11616-24. DOI

13. Wang Z, Cheng J, Guan Q, et al. All-in-one fiber for stretchable fiber-shaped tandem supercapacitors. Nano Energy 2018;45:210-9. DOI

14. Zhang L, Yang X, Zhang F, et al. Controlling the effective surface area and pore size distribution of sp2 carbon materials and their impact on the capacitance performance of these materials. J Am Chem Soc 2013;135:5921-9. DOI PubMed

15. Ma W, Li W, Li M, et al. Unzipped carbon nanotube/graphene hybrid fiber with less "dead volume" for ultrahigh volumetric energy density supercapacitors. Adv Funct Mater 2021;31:2100195. DOI

16. Qu G, Cheng J, Li X, et al. A fiber supercapacitor with high energy density based on hollow graphene/conducting polymer fiber electrode. Adv Mater 2016;28:3646-52. DOI PubMed

17. Jalili R, Razal JM, Innis PC, Wallace GG. One-step wet-spinning process of poly(3,4-ethylenedioxythiophene):poly(styrenesulfonate) fibers and the origin of higher electrical conductivity. Adv Funct Mater 2011;21:3363-70. DOI

18. Kim N, Kee S, Lee SH, et al. Highly conductive PEDOT:PSS nanofibrils induced by solution-processed crystallization. Adv Mater 2014;26:2268-72, 2109. DOI PubMed

19. Lang U, Müller E, Naujoks N, Dual J. Microscopical Investigations of PEDOT:PSS thin films. Adv Funct Mater 2009;19:1215-20. DOI

20. Vosgueritchian M, Lipomi DJ, Bao Z. Highly conductive and transparent PEDOT:PSS films with a fluorosurfactant for stretchable and flexible transparent electrodes. Adv Funct Mater 2012;22:421-8. DOI

21. Cai S, Huang T, Chen H, Salman M, Gopalsamy K, Gao C. Wet-spinning of ternary synergistic coaxial fibers for high performance yarn supercapacitors. J Mater Chem A 2017;5:22489-94. DOI

22. Xu D, Shen $\mathrm{H}$, Wang W, et al. Effect of $\mathrm{H}_{2} \mathrm{SO}_{4}$ solution treatment on adhesion, charge transfer, and catalytic performance of screenprinted PEDOT:PSS. Chemphyschem 2019;20:374-82. DOI

23. Li Y, Ren G, Zhang Z, et al. A strong and highly flexible aramid nanofibers/PEDOT:PSS film for all-solid-state supercapacitors with superior cycling stability. J Mater Chem A 2016;4:17324-32. DOI

24. Alemu D, Wei H, Ho K, Chu C. Highly conductive PEDOT:PSS electrode by simple film treatment with methanol for ITO-free polymer solar cells. Energy Environ Sci 2012;5:9662. DOI

25. Xu T, Yang D, Zhang S, Zhao T, Zhang M, Yu Z. Antifreezing and stretchable all-gel-state supercapacitor with enhanced capacitances established by graphene/PEDOT-polyvinyl alcohol hydrogel fibers with dual networks. Carbon 2021;171:201-10. DOI

26. Xu H, Hu X, Sun Y, Yang H, Liu X, Huang Y. Flexible fiber-shaped supercapacitors based on hierarchically nanostructured composite electrodes. Nano Res 2015;8:1148-58. DOI

27. Li B, Cheng J, Wang Z, Li Y, Ni W, Wang B. Highly-wrinkled reduced graphene oxide-conductive polymer fibers for flexible fibershaped and interdigital-designed supercapacitors. J Power Sources 2018;376:117-24. DOI

28. Sun P, Lin R, Wang Z, et al. Rational design of carbon shell endows TiN@C nanotube based fiber supercapacitors with significantly enhanced mechanical stability and electrochemical performance. Nano Energy 2017;31:432-40. DOI

29. Noh J, Yoon C, Kim YK, Jang J. High performance asymmetric supercapacitor twisted from carbon fiber/MnO $\mathrm{O}_{2}$ and carbon fiber/ $\mathrm{MoO}_{3}$. Carbon 2017;116:470-8. DOI

30. Kou L, Huang T, Zheng B, et al. Coaxial wet-spun yarn supercapacitors for high-energy density and safe wearable electronics. Nat Commun 2014;5:3754. DOI PubMed PMC

31. Sun G, Zhang X, Lin R, Yang J, Zhang H, Chen P. Hybrid fibers made of molybdenum disulfide, reduced graphene oxide, and multiwalled carbon nanotubes for solid-state, flexible, asymmetric supercapacitors. Angew Chem Int Ed Engl 2015;54:4651-6. DOI PubMed

32. Meng C, Qian Y, He J, Dong X. Wet-spinning fabrication of multi-walled carbon nanotubes reinforced poly(3,4ethylenedioxythiophene)-poly(styrenesulfonate) hybrid fibers for high-performance fiber-shaped supercapacitor. J Mater Sci: Mater Electron 2020;31:19293-308. DOI

33. Wang H, Wang C, Jian M, et al. Superelastic wire-shaped supercapacitor sustaining $850 \%$ tensile strain based on carbon nanotube@graphene fiber. Nano Res 2018;11:2347-56. DOI 